\title{
X-linked agammaglobulinaemia and squamous lung cancer
}

\author{
J.M. Echave-Sustaeta*, V. Villena*, M. Verdugo*, A. López-Encuentra*, \\ P. de Agustín ${ }^{\#}$, N. Alberti"
}

X-linked agammaglobulinaemia and squamous lung cancer. J.M. Echave-Sustaeta, V. Villena, M. Verdugo, A.López-Encuentra, P.de Agustín, N. Alberti. (C)ERS Journals Ltd 2001.

ABSTRACT: A 32 yr-old nonsmoking male, diagnosed as having X-linked agammaglobulinemia, presented with fever, cough with purulent sputum, a very intense back pain and a mass of 10 centimetres in lower left lobe. Diagnostic evaluation revealed a squamous cell carcinoma with very aggressive metastases at L3. Malignancies are the second leading cause of death in children and adults with congenital immunodeficiency disorders, mostly non-Hodgkin lymphomas and gastric and colon adenocarcinomas, but this is the first report of lung cancer in a patient with $X$-linked agammaglobulinemia. Lung cancer incidence has been reported to be higher in patients with other diseases of the lung, however, there is no clear evidence of the role of bronchiectasis in developing lung cancer. It is possible that a longer survival for patients with X-LA recently diagnosed, and an association of chronic bronchial infection, could favour the development of pulmonary neoplasm.

Eur Respir J 2001; 17: 570-572.

*Pneumology Service and ${ }^{\#}$ Pathology Dept, Doce de Octubre University Hospital, Madrid, Spain

Correspondence: J.M. EchaveSustaeta, Servicio de Neumología, Hospital Universitario Doce de Octubre, Crtra Andalucía km 5,4, 28041, Madrid, Spain.

Fax: $349146957 \quad 75$

Keywords: Bronchiectasis hypogammaglobulinaemia squamous lung cancer

$\mathrm{X}$-linked agammaglobulinaemia

Received: June 52000

Accepted after revision August 42000
Presented is a case of lung cancer arising in a patient with X-linked agammaglobulinaemia (X-LA). As far as the authors know it is the first case reported with this association.

\section{Case report}

A 32-yr-old nonsmoking male was referred to the authors' hospital because of the persistence of a lung abscess. He began, at the age of eighteen months, with frequent sinopulmonary infections. When he was 3 yrs old he suffered pneumonia, and at 4 yrs an arthritis in hip. At that time he was diagnosed with X-LA. The diagnosis was performed by a combination of an absence of circulating mature B-cells, profoundly reduced levels of serum immunoglobulin (Ig)G, IgM, $\operatorname{IgA}$, and normal T-cells count. Initially, replacement with intramuscular Igs was attempted which led to improvements in clinic status. However, acute infections persisted and chronic infections developed, particularly chronic sinusitis and bronchitis leading to bronchiectasis and chronic obstructive lung disease. In 1985 the treatment was changed to intravenous Ig, and the incidence of bacterial and nonbacterial infections was significantly reduced, with also an improvement in their course. In 1987 he was diagnosed of amyloid goiter, and in 1988 a partial thyroidectomy was carried out, with a post-surgery hypothyroidism. In 1995 he developed a chronic renal failure with a creatinin clearance of $35 \mathrm{~mL} \cdot \mathrm{h}^{-1}$, probably due to use of aminoglycoside antibiotic.
Four months before admission, he was treated at another hospital because of fever, sweats, cough and the presence of a mass at lower left lobe. A sputum culture showed Pseudomona Aeruginosa. He improved initially with a combination of ceftazidime and ciprofloxacine, but the chest radiograph did not change. He was transferred to the authors' hospital to perform a diagnostic procedure.

On admission, in February 1999, the patient referred fever, cough with copious purulent sputum and a very intense back pain, which did not improve with acetaminophen. The white-cell count was 14,670 per cubic millimeter, and the haematocrit 30.5\%. The erythrocyte sedimentation rate was $70 \mathrm{~mm} \cdot \mathrm{h}^{-1}$. Radiographs of the chest showed a mass of $10 \mathrm{~cm}$ in lower left lobe. A computer tomographic scan of the chest confirmed a mass of $10 \mathrm{~cm}$ in lower left lobe and multiple dilated bronchi characteristic of bronchiectasis (fig. 1). Pulmonary function studies showed a severe obstructive limitation, with a forced expiatory volume in one second (FEV1) of $0.85 \mathrm{~L}(23 \%)$.

A fibreoptic bronchoscopic examination showed purulent secretions. Bronchial brushing and transbronchial biopsy from lower left lobe were done. The transbronchial biopsy revealed bronchial mucosae with acute and chronic unspecific inflammation, and the bronchial brushing was positive for squamous cell carcinoma. A percutaneous fine needle aspiration cytology (FNAC) of the mass under computed tomography (CT) guidance confirmed the diagnosis of squamous cell carcinoma (fig. 2). A radionuclide bone scan showed an area of increased uptake in 


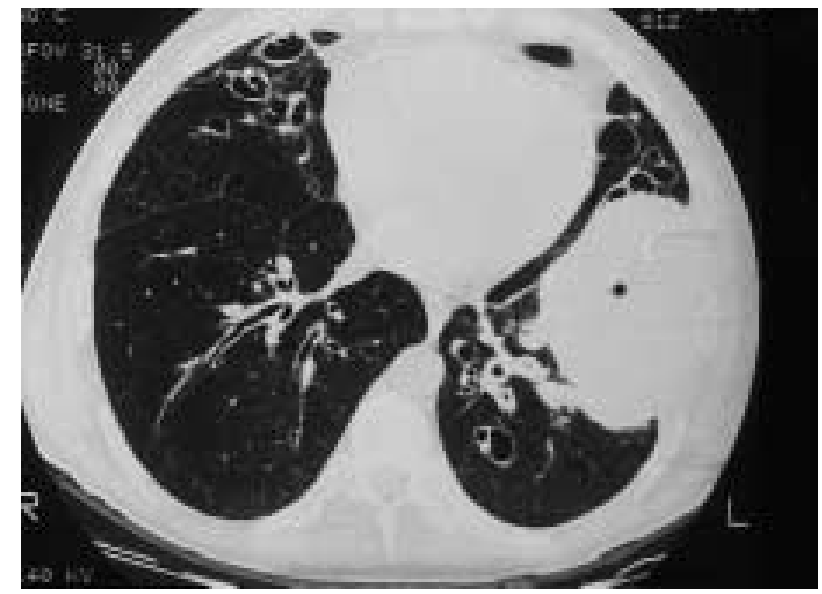

Fig. 1. - Computer tomographic scan of the chest showing a mass of $10 \mathrm{~cm}$ in lower left lobe and multiple dilated bronchi characteristic of bronchiectasis.

lumbar spine in L3 and L4 characteristic of metastases. A nuclear magnetic resonance (NMR) of the lumbar vertebrae showed very aggressive metastases with epidural and medullar compromise at L3 (fig. 3).

During hospital stay the patient needed very intensive analgesia. The diagnosis of primary tumour, regional node, metastasis (TNM) stage IV (M1) squamous lung cancer was assumed. The patient began with symptoms of spinal compression and palliative radiation therapy was immediately started with a total dose of 38 Gy $(10 \times 3 \mathrm{~Gy})$ from L2 to L5, with good tolerance and improvement of the symptoms. The patient was discharged and died two months later.

\section{Discussion}

X-LA, sometimes termed Bruton disease, was first described nearly 50 yrs ago, and remains the prototypic syndrome of a pure B-cell deficiency [1]. The disorder has a relatively homogeneous clinical presentation. Affected males are usually well for the first 9-12 months of life because they are passively protected by transplacentally acquired IgG from their mothers. Subsequently, they have recurrent pyogenic infections. However, $40 \%$ of patients remain asymptomatic in their first year of life, with $21 \%$ presenting between the ages of 3-5 yrs [2]. The gene encoding the cytoplasmic Bruton tyrosine kinase, map to the long arm of the $\mathrm{X}$-chromosome at position Xq21.2-22, is defective in $\mathrm{X}$-LA. In the Spanish Registry for Primary Immunodeficiency Diseases, with 1,069 cases registered between January 1980 and December 1995, 49 (4.6\%) of them were X-LA, and the incidence per million live births for the X-LA during the period 1990-1994 was 0.05 [3].

Reports of tumours arising in patients with immunodeficiency disorders have appeared in increasing numbers in recent decades. After infections, malignancies are the second leading cause of death in children and adults with congenital immunodeficiency disorders [4]. The higher incidence of neoplasms observed in patients with primary immunodeficiency diseases is

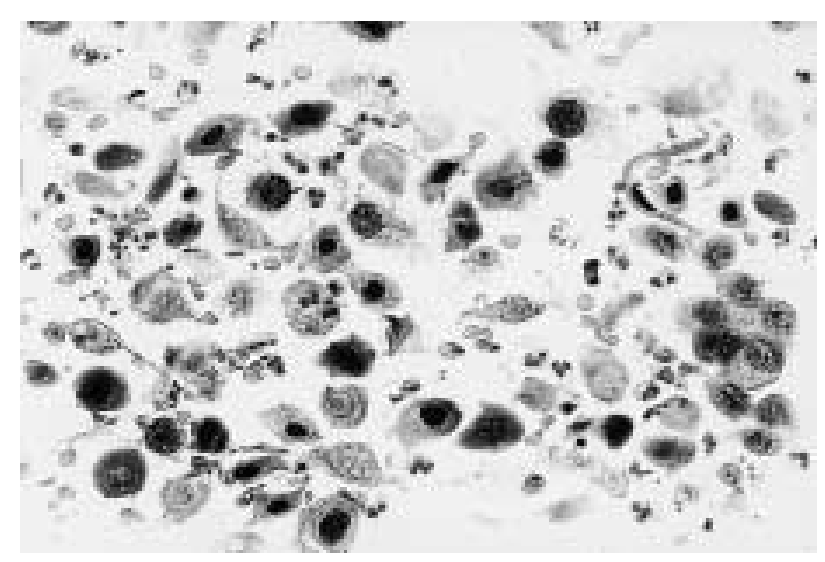

Fig. 2. - Squamous cell carcinoma of the lung. Numerous dissociated tumour cells that have dense cytoplasm with endo and ectoplasm zones and hyperchromatic nuclei. Note one spindleshaped cell in the right upper corner and the cellular necrosis appearing as ghost cells (original magnification) (internal scale bar $=40 \mu \mathrm{m})$.

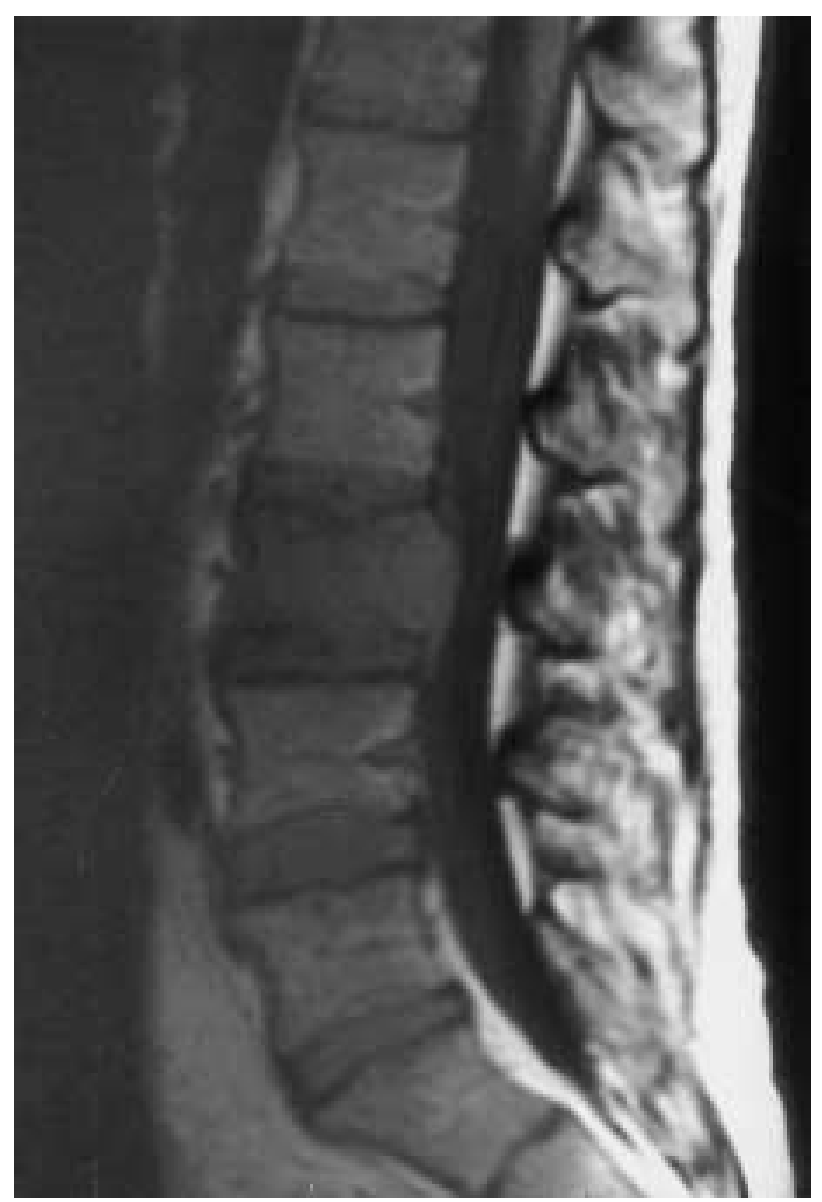

Fig. 3. - Nuclear magnetic resonance of the lumbar vertebrae showing very aggressive metastases with epidural and medullar compromise at L3.

probably a consequence of the increased survival of these patients, because of the standard use of intravenous gamma globulin and a better management of recurrent infections [5]. Different reports quotes a $1.5-2 \%$ risk for the development of malignancy among 
patients with X-LA $[3,4,6,7]$. It is notable that a relatively limited repertoire of cancers has been observed, mostly non-Hodgkin lymphomas and gastric and colon adenocarcinomas $[3-5,7,8]$. The immunological abnormalities that predispose patients with lymphoma are defects in immunoregulation and an inability to eradicate viral infections [5]. Immune impairment itself does not appear to be a risk factor for gastric or colon adenocarcinoma because other groups of immunodeficient patients, such as those taking immunosupresive drugs, show no excess of this malignancy. In the gastric and colon carcinoma it is possible that an increased susceptibility to any pathogens present in the stomach or gut may lead to chronic inflammation and dysplasia $[5,8]$. Some studies have shown a strong association between Helicobacter pylori infection with chronic gastritis and gastric adenocarcinoma. In these agammaglobulinaemic patients, the risk of early gastric infection by this bacteria is probably increased. It has been hypothesized that the risk of gastric adenocarcinoma is dependent on the precocity of the infection, which might provide sufficient time for multiple mutations and malignant transformation [9].

The first case of lung cancer in a patient with X-LA is reported, although one case of squamous cell lung cancer has been described in a patient with noninvasive thymoma and severe hypogammaglobulinaemia [10]. The first generation of patients with X-LA who reach adulthood are now being seen. In a recent report nearly $60 \%$ of patients with X-LA were alive at $30 \mathrm{yrs}$, and there is a trend towards better survival for patients recently diagnosed [2]. What pathogenic mechanisms could underlay the development of the lung cancer in the presented patient? Lung cancer incidence has been reported to be higher in patients with other diseases of the lung, such as chronic obstructive lung disease, tuberculosis, chronic bronchitis, or silicosis, that could retard mucociliary clearance of foreign particles, evoking an inflammatory response, and causes mucous gland hypertrophy, hyperplasia, and dysplasia in proximal airways. The link between pulmonary tumourlets and microcarcinoids in bronchiectasis is well known, and recent evidence also suggests a connection between cellular immunodeficiency such as the human immunodeficiency virus infection and the lung cancer. However, there is no clear evidence of the role of bronchiectasis in developing lung cancer. Some reports maintain that nodular lung carcinomas could arise from a cluster of nonobstructive bronchiectasis. Tonelli [11] suggests that lung carcinomas occurring in bronchiectasis of this kind are usually peripheral, nodular, sharply outlined, sometimes excavated, and in high incidence epidermoid. As for all lung carcinomas, cigarette smoking also plays a prominent role in aetiopathogenesis. The presented case shares most of these characteristics, but the patient was not a smoker. Maybe, as with gastric and colon carcinomas, an association between chronic bronchial infection and multiple mutations and malignant transformation is present. No other explanation for the tumour could be found.

In conclusion, this is the first case report of a patient with X-linked agammaglobulinaemia, diffuse bronchiectasis and a lung carcinoma. It is possible that with a longer survival for patients with X-linked agammaglobulinaemia recently diagnosed, in the future more patients with this association could be seen.

\section{References}

1. Rosen FS, Cooper MD, Wedgwood RJP. The primary immunodeficiencies. N Engl J Med 1995; 333: $431-$ 440.

2. Hermaszewski RA, Webster ADB. Primary hypogammaglobulinemia: a survey of clinical manifestations and complications. $Q$ J Med 1993; 86: $31-42$.

3. Matamoros Flori N, Mila Llambi J, Español Boren T, Raga Borja S, Fontan Casariego G. Primary immunodeficiency syndrome in Spain: first report of the national registry in children and adults. $J$ Clin Immunol 1997; 17: 333 - 339.

4. Mueller BU, Pizzo PA. Cancer in children with primary or secondary immunodeficiencies. J Pediatr 1995; 126: $1-10$.

5. Lavilla $\mathrm{P}$, Gil AG, Rodríguez MC, Dupla ML, Pintado V, Fontán G. X-linked agammaglobulinemia and gastric adenocarcinoma. Cancer 1993; 72: $1528-1531$.

6. Lederman HM. Cancer in children with primary or secondary immunodeficiencies. J Pediatr 1995; 127: 335.

7. Zenone T, Souillet G. Cancer et déficit primitif de l'immunité humorale. Bull Cancer 1997; 84: 813-821.

8. Van der Meer JWM, Weening RS, Schellekens PTA, van Munster IP, Nagengast FM. Colorectal cancer in patients with X-linked agammaglobulinemia. Lancet 1993; 341: 1439-1440.

9. Parsonnet J, Friedman GD, Vandersteen DP, et al. Helicobacter pylori infection and the risk of gastric adenocarcinoma. $N$ Engl J Med 1991; 325: 1127-1131.

10. Montresor E, Falezza G, Vassia S, et al. Thymoma, aplastic anemia, hypogammaglobulinemia and malignant pulmonary neoplasm: a case report. G Chir 1988; 19: $92-95$.

11. Tonelli P. A morphologic study of nodular lung carcinomas and their possible pathogenesis from a cluster of non-obstructive bronchiectasis. Lung-Cancer 1997; 17: 135-145. 